\title{
Finetuning the continuum limit in low-dimensional supersymmetric theories *
}

\author{
Bjoern H. Wellegehausen \\ Justus-Liebig-University Giessen \\ E-mail: bjoern.wellegehausen@theo.physik.uni-giessen.de

\section{Daniel Koerner ${ }^{\dagger}$ and Andreas Wipf} \\ Friedrich-Schiller-University Jena \\ E-mail: daniel.koerner@uni-jena.de, wipf@tpi.uni-jena.de
}

\begin{abstract}
Supersymmetry is a prominent candidate for physics beyond the standard model. In order to compute the spectrum of supersymmetric theories, we employ nonperturbative lattice QFT techniques which due to the discretisation of spacetime violate supersymmetry at finite lattice spacings. Care has to be taken then to restore supersymmetry in the continuum limit. We discuss a discretisation of the supersymmetric Nonlinear $\mathrm{O}(\mathrm{N})$ Sigma model in two dimensions and argue that supersymmetry may be restored by finetuning of a single parameter. Furthermore, we show preliminary results for the vacuum physics of $\mathscr{N}=2$ Super-Yang-Mills theory in three dimensions.
\end{abstract}

31st International Symposium on Lattice Field Theory LATTICE 2013

July 29 - August 3, 2013

Mainz, Germany

${ }^{*}$ We thank Raphael Flore and Christian Wozar for their active collaboration and Georg Bergner and Mithat Ünsal for helpful discussions.

${ }^{\dagger}$ Speaker. 


\section{Beyond the Standard Model - Supersymmetry}

The Standard Model provides a very successful description of electroweak and strong interactions. However, cosmological observations uncover discrepancies from the theoretical prediction, i.e. the matter to antimatter ratio or dark matter. These observations point to physics beyond the Standard Model. Supersymmetry, an extension of the Poincaré symmetry of spacetime transformations, provides a possible extension of the Standard model. The algebra of supercharges closes on the energy-momentum,

$$
\left\{Q_{\alpha}, \bar{Q}_{\beta}\right\}=2 \gamma_{\alpha \beta}^{\mu} P_{\mu} .
$$

On a discrete spacetime lattice, infinitesimal translations or rotations are not possible and hence supersymmetry is inevitably broken at finite lattice spacing. In order to restore supersymmetry in the continuum limit, all relevant susy-breaking operators need to be controlled. A recent approach [1] tries to implement a part of the supersymmetry exactly on the lattice, which may guarantee the correct continuum limit. However, we will show that this approach fails for the supersymmetric nonlinear Sigma Model and we then proceed to argue that a finetuning of susy-breaking operators leads to the expected continuum limit. Furthermore we show that for $\mathscr{N}=2$ supersymmetric Yang-Mills theory a supersymmetric continuum limit is possible by finetuning a single parameter.

\section{Supersymmetric $\mathbf{O}(3)$ nonlinear $\sigma$ model}

The supersymmetric extension of the bosonic $\mathrm{O}(3)$ nonlinear Sigma model is formulated most elegantly in superspace, where the usual constraint is applied to the superfield. To obtain the action in regular spacetime, we expand the superfield and integrate out the auxiliary field with the result

$$
S=\frac{1}{2 g^{2}} \int d^{2} x\left(\partial^{\mu} \mathbf{n} \partial_{\mu} \mathbf{n}+i \bar{\psi} \not \partial \psi+\frac{1}{4}(\bar{\psi} \psi)^{2}\right), \quad \text { where } \mathbf{n}^{2}=1, \quad \mathbf{n} \psi=0 .
$$

In order to simulate the theory on the lattice, it is necessary to rewrite the contraints for the component fields. We choose a stereographic projection of the superfield which resolves the field constraints explicitly [2]. In addition to supersymmetry, the classical action is invariant under $\mathrm{SO}(3)$-rotation of the fields. Furthermore, a discrete $\mathbb{Z}_{2}$ chiral symmetry is broken spontaneously on the lattice for arbitrary coupling and is connected to the dynamical generation of mass.

Supersymmetry relates the bosonic and fermionic components of the superfields,

$$
\delta_{I} n_{i}=i \bar{\varepsilon} \psi_{i}, \quad \delta_{I} \psi_{i}^{\alpha}=\not \partial n_{i} \varepsilon+\frac{i}{2}(\bar{\psi} \psi) n_{i} \varepsilon^{\alpha} .
$$

Since the target space $S^{2}$ is Kähler, there exists an $\mathscr{N}=2$-supersymmetric extension of the model. The second susy transformation reads

$$
\delta_{I I} n=n \times i \bar{\varepsilon} \psi, \quad \delta_{I I} \psi^{\alpha}=-n \times(\not \partial n \varepsilon)^{\alpha}-i \bar{\varepsilon} \psi \times \psi^{\alpha} .
$$

The supersymmetries are generated by the supercharges:

$$
Q_{\alpha}^{I}=\int i \partial_{\mu} n_{i} \gamma^{\mu} \gamma^{0} \psi_{i}, \quad Q_{\alpha}^{I I}=\int-i \varepsilon_{i j k} n_{i} \partial_{\mu} n_{j} \gamma^{\mu} \gamma^{0} \psi_{k}
$$



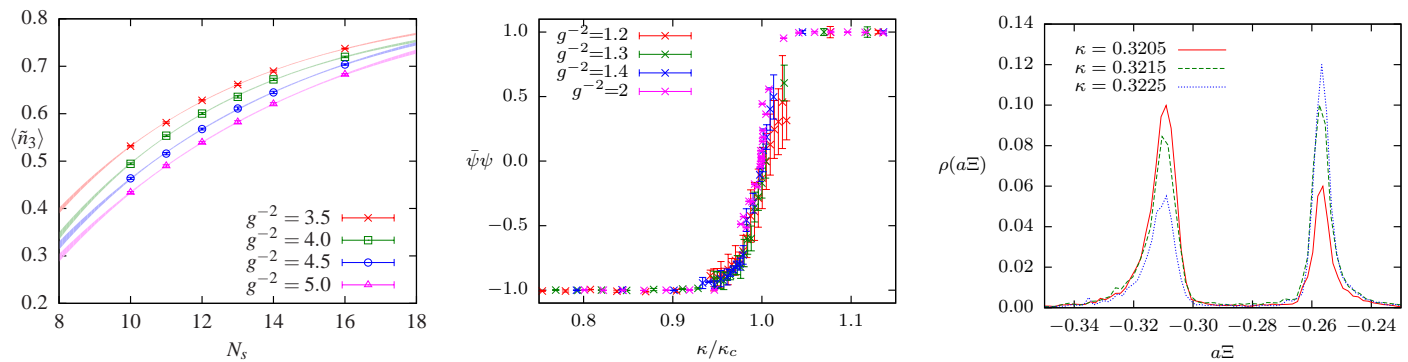

Figure 1: Left panel: measurement of the order parameter $\left\langle n_{3}\right\rangle$ in the $Q$-exact ensemble for different coupling constants and lattice volumes. Center panel: phase transition of the renormalized chiral condensate at the critical finetuning parameter $\kappa_{c}$. Right panel: $\mathbb{Z}_{2}$-symmetric histogram of the chiral condensate.

\subsection{Implementing exact supersymmetry and $O(3)$ symmetry - a no-go theorem}

The authors of [3] construct a nilpotent supercharge $Q^{2}=0$, such that the action may be written as $S=Q \Lambda$. By implementing this relation exactly on the lattice, part of the supersymmetry is respected and the supersymmetric continuum limit may be approached without the need for finetuning. However, this " $Q$-exact action" breaks the $\mathrm{O}(3)$ symmetry explicitly at any lattice spacing. Mermin-Wagner's theorem dictates that the $\mathrm{O}(3)$ symmetry cannot be broken spontaneously and one therefore needs to take care in restoring the symmetry in the continuum limit. Alas, by simulating the action of [3] we find that the order parameter of the $\mathrm{O}(3)$ symmetry does not vanish in the infinite volume limit, as is shown in Fig. 1 (left panel), and the resulting continuum limit does not belong to the supersymmetric O(3) NLSM, therefore rendering the $Q$-exact discretisation invalid [2]. This failure raises the question whether it is possible at all to find a lattice formulation of this model that preserves both the $\mathrm{O}(3)$ symmetry as well as part of supersymmetry. We will argue that this is indeed not possible. A general symmetry of the model needs to leave invariant both the action as well as the constraints. The first susy transformation in eq. 2.2 breaks the constraint $n \psi=0$ at finite lattice spacing,

$$
\delta_{I}\left(n_{x} \psi_{x}^{\alpha}\right)=i \bar{\varepsilon} \psi_{x} \psi_{x}^{\alpha}+\sum_{y \in L} n_{x} D_{x y}^{\alpha \beta} n_{y} \varepsilon^{\beta}+\frac{i}{2}\left(\bar{\psi}_{x} \psi_{x}\right) n_{x}^{2} \varepsilon^{\alpha}=\sum_{y \in L} n_{x} D_{x y}^{\alpha \beta} n_{y} \varepsilon^{\beta}
$$

The second susy transformation always respects the constraints by use of vector identities,

$$
\delta_{I I}\left(n_{x} \psi_{x}\right)=\sum_{y \in L} n_{x} \cdot\left(n_{x} \times D_{x y}^{\alpha \beta} n_{y} \varepsilon^{\beta}\right)=0, \quad \delta_{I I}\left(n_{x}^{2}\right)=2 n_{x}\left(n_{x} \times i \bar{\varepsilon} \psi_{x}\right)=0 .
$$

However, the second susy transformation by itself cannot be a symmetry on the lattice since the susy algebra cannot be closed (eq. 1.1) and any nontrivial combination of the susy transformations $\delta_{I}$ and $\delta_{I I}$ is not compatible with the constraints. The approach in [3] contains an additional topological charge, which however does not alter the supersymmetry transformations and thus the problem persists. One may further introduce nonlocal interaction terms like $\sum_{y, z, w} C_{x y z w} \psi_{x} \psi_{y} \psi_{z} \psi_{w}$ instead of $\left(\psi_{x}\right)^{4}$. But none of such terms is able to cure the problem at hand. The last remaining possibility is the introduction of terms that vanish in the continuum limit, but render the lattice action invariant under $Q^{I I}$ for finite lattice spacing. Once again, no appropriate terms are available. 

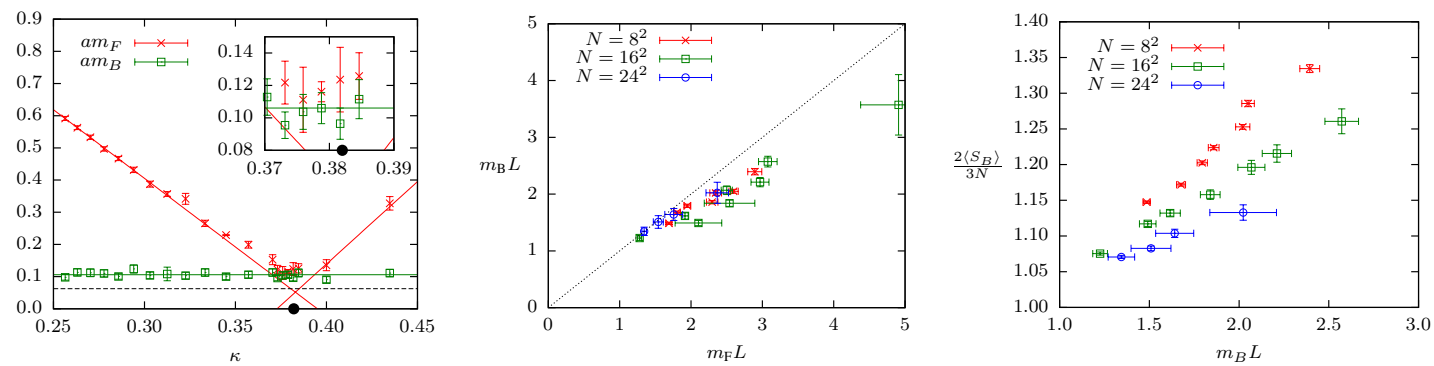

Figure 2: Left panel: finetuning $\kappa$ towards the critical value $\kappa_{c}$ reveals a possible mass degeneracy. Center panel: bosonic and fermionic mass gap for Wilson fermions at $\kappa=\kappa_{c}$. Right panel: keeping the box size $m_{B} L$ fixed, the simple Ward identity is restored in the continuum limit.

We thus conclude that no discretization of the $\mathrm{O}(3)$-NLSM exists which maintains $\mathrm{O}(3)$-invariance and exact supersymmetry.

\subsection{Finetuning the supersymmetric continuum limit}

In order to discretize the fermionic degrees of freedom, we replace the continuum Dirac operator by the Wilson lattice operator which suppresses unwanted fermion doublers. While being ultralocal, the Wilson operator introduces an explicit breaking of chiral symmetry which should be cancelled by finetuning the hopping parameter $\kappa$. In figure 1 (center panel) we show the first order phase transition of the renormalized chiral condensate which marks the critical value $\kappa_{c}$. At $\kappa=\kappa_{c}$, we can cleary distinguish the two minima of the chiral condensate in the right panel of figure 1. Supersymmetry predicts that the masses of the elementary bosonic and fermionic excitations coincide in the continuum limit. Adjusting the hopping parameter as in figure 2 (left panel), we see that the bosonic mass stays constant when we vary $\kappa$, whereas the fermionic mass shows a linear dependence in a large range. In the vicinity of $\kappa_{c}$, this linear dependence ceases and the fermionic mass does not go to zero but stays at the level of the bosonic mass, hinting at a possible degeneracy. Using the finetuned ensemble in figure 2 (center panel) we find a residual discrepancy of the masses for intermediate box sizes $m_{F} L$, which can however be attributed to a thermal contribution to the mass gap. A similar behaviour can be seen in the Wess-Zuminomodel [4] and we expect it to vanish for large box sizes. The bosonic action constitutes a simple Ward identity for susy restoration, $\left\langle S_{B}\right\rangle=\frac{3}{2} N$, and we find that the relation is restored in the continuum limit (see right panel of Fig. 2). Both the observed masses and the Ward identity show a restoration of supersymmetry when $\kappa$ is finetuned.

\section{3. $\mathscr{N}=1$ supersymmetric Yang-Mills theory in 4 dimensions}

The on-shell action in four spacetime dimensions is given by $[5,6]$

$$
S_{\mathrm{SYM}}=\int d^{4} x \operatorname{tr}\left\{-\frac{1}{4} F_{\mu v} F^{\mu v}+\frac{i}{2} \bar{\lambda} \gamma_{\mu} D^{\mu} \lambda+m \bar{\lambda} \lambda\right\}
$$

where $F_{\mu \nu}=\partial_{\mu} A_{v}-\partial_{v} A_{\mu}-i g\left[A_{\mu}, A_{v}\right]$ is the usual field strength tensor with dimensionless gauge coupling constant $g$. The gauge field $A_{\mu}$ and the Majorana spinor $\lambda$ transforms under the adjoint 
representation of the gauge group $S U\left(N_{\mathrm{c}}\right)$. For $m=0$ the action (3.1) is invariant under $\mathscr{N}=1$ supersymmetry transformations

$$
\delta A_{\mu}=\mathrm{i} \bar{\varepsilon} \gamma_{\mu} \lambda \quad, \quad \delta \lambda=\mathrm{i} \Sigma_{\mu \nu} F^{\mu v} \varepsilon \quad \text { and } \quad \delta \bar{\lambda}=-\mathrm{i} \bar{\varepsilon} \Sigma_{\mu v} F^{\mu v},
$$

where $\varepsilon$ is an arbitrary constant Majorana spinor. The gluino mass term $m$ introduces a soft breaking of supersymmetry. The action (3.1) is invariant under a chiral $U(1)_{\mathrm{A}}$ transformation (Rsymmetry) that is broken by the chiral anomaly to the discrete subgroup $\mathbb{Z}\left(2 N_{\mathrm{c}}\right)$. A non-vanishing gluino condensate $\langle\bar{\lambda} \lambda\rangle \neq 0$ breaks the discrete symmetry further down to $\mathbb{Z}\left(N_{\mathrm{c}}\right)$, leaving $N_{\mathrm{c}}$ inequivalent ground states of the theory. For $N_{\mathrm{c}}=2$, two degenerate ground states should exist, that can be distinguished by the sign of the gluino condensate. It is expected that the theory possesses a first order phase transition at vanishing renormalized gluino mass, if the chiral symmetry is spontaneously broken [7]. In [5], Veneziano and Yankielowicz argued, that the only supersymmetry breaking operator is related to a non-vanishing gluino condensate. In order to restore supersymmetry on the lattice, it is therefore sufficient to fine-tune the theory to a massless gluino in the continuum limit. Due to confinement, the gluino is not part of the physical spectrum, and it is not possible to measure its mass directly. But the OZI rule (known from QCD) relates the renormalized gluino mass to the pion mass as $m_{g} \propto m_{\pi}^{2}$ [8]. Very recently, $\mathscr{N}=1$ SYM theory in four dimensions has been investigated with much effort on the lattice [8-12]. But so far the results for the mass spectrum are not conclusive.

As in Yang-Mills theories, it is believed that in SYM theories only colourless asymptotic states exist and a mass gap is dynamically generated. Veneziano and Yankielowicz (VY) [5] and later Farrar-Gabadadze-Schwetz (FGS) [13] proposed an effective Lagrangian, that leads to the particle spectrum shown in Table 1. If supersymmetry is broken by a gluino mass term, the masses inside

\begin{tabular}{c|lccccc} 
multiplet & particle & operator & spin & mass & SYM-name & QCD-name \\
\hline \hline \multirow{3}{*}{ VY } & 1 pseudoscalar boson & $\operatorname{tr} \bar{\lambda} \gamma_{5} \lambda$ & 0 & $m_{\tilde{g} \tilde{g}}^{0-}$ & $a-\eta^{\prime}$ & $\eta^{\prime}$ \\
& 1 scalar boson & $\operatorname{tr} \bar{\lambda} \lambda$ & 0 & $m_{\tilde{g} \tilde{g}}^{0+}$ & $a-f_{0}$ & $f_{0}$ \\
& 1 Majorana fermion & $\operatorname{tr} F_{\mu v} \Sigma^{\mu v} \lambda$ & $\frac{1}{2}$ & $m_{g \tilde{g}}$ & gluino-glueball & - \\
\hline \multirow{3}{*}{ FGS } & 1 scalar boson & $\operatorname{tr} F^{\mu v} F_{\mu v}$ & 0 & $m_{g g}^{0+}$ & $0^{+}-$glueball & $0^{+}$- glueball \\
& 1 pseudoscalar boson & $\operatorname{tr} F^{\mu v} \tilde{F}_{\mu v}$ & 0 & $m_{g g}^{0-}$ & $0^{-}-$glueball & $0^{-}$- glueball \\
& 1 Majorana fermion & $\operatorname{tr} F_{\mu v} \Sigma^{\mu v} \lambda$ & $\frac{1}{2}$ & $m_{g \tilde{g}}$ & gluino - glueball & -
\end{tabular}

Table 1: Particles of the Veneziano-Yankielowicz (VY) and Farrar-Gabadadze-Schwetz (FGS) multiplet.

one multiplet are no longer degenerate and the $0^{+}$glueball should be the lightest particle.

\section{4. $\mathscr{N}=2$ supersymmetric Yang-Mills theory in 3 dimensions}

Here we investigate the theory in a dimensional reduced version in three dimensions. The action for this (euclidean) $\mathscr{N}=2$ supersymmetric Yang-Mills theory is then given by ${ }^{1}$

$$
S^{\mathrm{E}}=\alpha \int d^{3} x \operatorname{tr}\left\{\frac{1}{4} F_{I J} F_{I J}+\frac{1}{2} \bar{\lambda} \gamma_{I}^{\mathrm{E}} D_{I} \lambda+\frac{1}{2} D_{I} \phi D_{I} \phi-\frac{1}{2} \bar{\lambda} \gamma_{3}^{\mathrm{E}}[\phi, \lambda]+m \bar{\lambda} \lambda\right\} .
$$

\footnotetext{
${ }^{1}$ We consider only a gluino mass term to fine-tune the theory, since we expect a scalar mass term to be small in the continuum limit. Nevertheless we have to compare our results to the effective scalar potential in [14].
} 
On a $16^{2} \times 32$ lattice for Wilson fermions, for different values of the overall gauge coupling $\alpha$, the critical hopping parameter $\kappa_{\mathrm{c}, \mathrm{OZI}}(\alpha)$ is determined such that the gluino becomes massless. In Fig. 3 (left panel) the square of the pion mass is shown for $\alpha=2.2$. A linear fit to the gluino mass yields
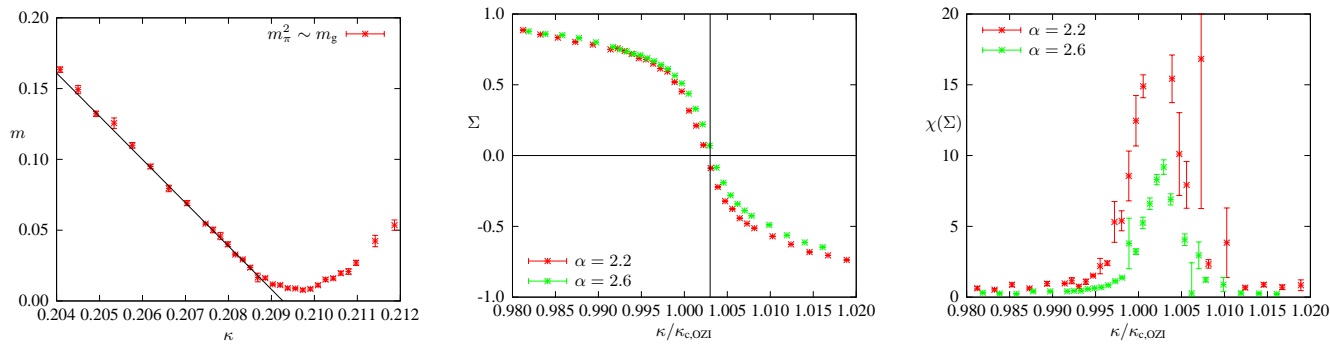

Figure 3: Gluino mass (square of the pion mass) on a $16^{2} \times 32$ lattice for $\alpha=2.2$ (left panel). The black lines represent a linear fit to the limit of a vanishing gluino mass. The Renormalized chiral condensate (center panel) and the chiral susceptibility (right panel) are shown for $\alpha=2.2$ and $\alpha=2.6$.

$\kappa_{\mathrm{c}, \text { OZI }}$ in Tab. 2. Due to the residual Wilson mass at a finite lattice spacing, the chiral condensate is renormalized as $\Sigma_{\text {ren }}(\alpha, \kappa)=Z_{1}\left(\Sigma(\alpha, \kappa)-m_{\text {res }}\right)=Z_{1} \Sigma(\alpha, \kappa)-Z_{2} \kappa-Z_{3}$, where it is assumed that the residual Wilson mass is a linear function in $\kappa$ (as it is the gluino mass). The renormalization constants $Z_{1}, Z_{2}$ and $Z_{3}$ are fixed such that $\Sigma_{\text {ren }}\left(\alpha, \kappa \ll \kappa_{c}\right)=1=-\Sigma_{\text {ren }}\left(\alpha, \kappa \gg \kappa_{c}\right)$. The critical point obtained from the vanishing of the pion mass can be compared to the critical point obtained from the transition in the chiral condensate. Therefore in the center and right panel of Fig. 3 the chiral condensate and its susceptibility are shown as a function of $\kappa$, normalized to the critical $\kappa_{\mathrm{c}, \text { OZI }}(\alpha)$ obtained before. For both $\alpha=2.2$ and $\alpha=2.6$, the deviations in the critical couplings are
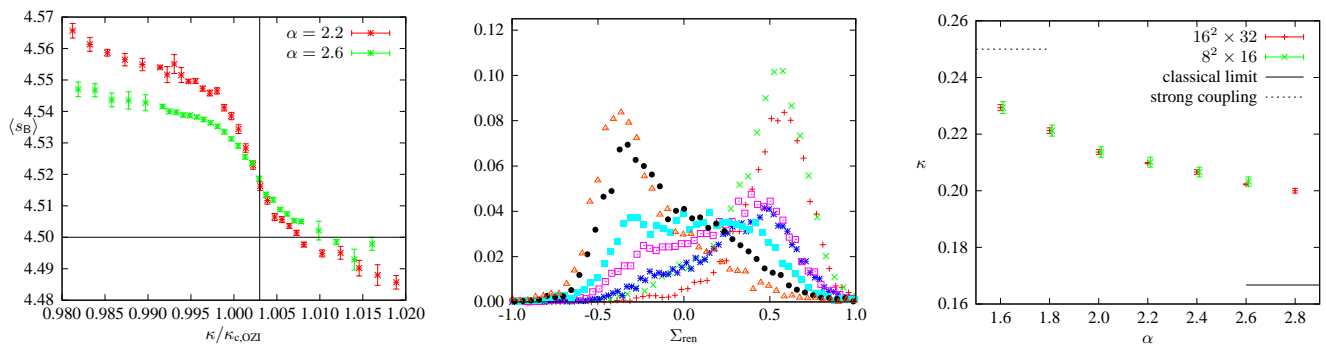

Figure 4: The bosonic action (left panel) is shown for $\alpha=2.2$ and $\alpha=2.6$. The Histograms of the chiral condensate in the center panel point to a first oder phase transition as a function of the renormalized gluino mass. In the right panel the critical line is shown.

less than 0.5 percent, see Tab. 2. In the left panel of Fig. 4 the bosonic action is plotted. Both curves

\begin{tabular}{c|cc}
$\alpha$ & $\kappa_{\mathrm{c}, \mathrm{OZI}}$ & $\kappa_{\mathrm{c}}$ \\
\hline \hline 2.2 & $0.2092(1)$ & $0.2097(4)$ \\
2.6 & $0.20167(2)$ & $0.2023(2)$
\end{tabular}

Table 2: $\kappa_{c}$ obtained from the vanishing gluino mass (left) and from the chiral condensate (right).

for $\alpha=2.2$ and $\alpha=2.6$ intersect each other almost exactly at the critical coupling obtained from 
the chiral condensate. The deviation from the theoretical value for restoration of supersymmetry at the intersection point is also about 0.5 percent, indicating that a restoration of supersymmetry on the lattice for this model is possible. As pointed out before, the chiral condensate as a function of $\kappa$ should undergo a first order phase transition at the point where the gluino mass vanishes. Therefore, in Fig. 4 (center panel) histograms of the chiral condensate for $\alpha=2.2$ are shown in the vicinity of the critical coupling. The double peak structure and the coexistence of both phases at the critical coupling clearly point to a first order transition. This indicates that chiral symmetry is spontaneously broken in the theory and supersymmetry can be restored in the continuum limit, i.e. the soft breaking of supersymmetry due to the Wilson mass can be removed by fine-tuning the bare gluino mass. In order to determine the critical line in the $(\alpha, \kappa)$-plane, the above sketched analysis is performed for different values of the gauge coupling and on different lattices. The results for two different lattices $8^{3} \times 16$ and $16^{3} \times 32$ are shown in Fig. 4 (right panel).

\section{Conclusions and Outlook}

In the first part we have shown that it is not possible to obtain a discretisation for the O(3) NLSM that preserves both $\mathrm{O}(3)$ symmetry and part of supersymmetry on the lattice. Our formulation incorporates the $\mathrm{O}(3)$ symmetry exactly but breaks supersymmetry. We argued that a finetuning of the hopping parameter is sufficient to cancel the explicit breaking of chiral symmetry.

For $\mathscr{N}=2$ Super-Yang-Mills theory in three dimensions we showed explicitly for the gauge group $S U(2)$ that it is possible to perform a supersymmetric continuum limit by finetuning the theory to a vanishing renormalized gluino mass. Our next aim is to investigate the spectrum of bound states as predicted by the effective lagrangian. However, this is difficult due to the involved disconnected correlation functions which typically show a very low signal-to-noise ratio.

\section{References}

[1] S. Catterall, D. Kaplan and M. Unsal, Phys. Rept. 484 (2009) 71-130.

[2] R. Flore, D. Körner, A. Wipf and C. Wozar, JHEP 11 (2012) 159.

[3] S. Catterall and S. Ghadab, JHEP 10 (2006) 063.

[4] T. Kaestner, G. Bergner, S. Uhlmann, A. Wipf and C. Wozar, Phys. Rev. D 78 (2008) 095001.

[5] G. Veneziano and S. Yankielowicz, Phys. Lett. B 113 (1982) 231 - 236.

[6] S. Ferrara, B. Zumino, and J. Wess, Phys. Lett. B 51 (1974) 239 - 241.

[7] R. Kirchner, I. Montvay et al. Phys. Lett. B 446 (1999) 209-215.

[8] A. Donini, M. Guagnelli, P. Hernandez, and A. Vladikas, Nucl. Phys. B 523 (1998) 529-552.

[9] G. Curci and G. Veneziano, Nucl. Phys. B 292 (1987) 555.

[10] G. Bergner, I. Montvay, G. Munster, D. Sandbrink, and U. D. Ozugurel, [arXiv:1111.3012].

[11] F. Farchioni and R. Peetz, Eur. Phys. J. C 39 (2005) 87-94.

[12] J. Giedt, R. Brower, S. Catterall, G. Fleming and P. Vranas, Phys. Rev. D 79 (2009) 025015.

[13] G.R. Farrar, G. Gabadadze, and M. Schwetz, Phys. Rev. D 58 (1998) 015009.

[14] Mithat Unsal, Phys.Rev. D80:065001, 2009. 